\title{
A Comparison of Hybrid Decision Making Methods for Emergency Support
}

\author{
Ángel Iglesias \\ M. Dolores del Castillo \\ J. Ignacio Serrano \\ Jesús Oliva \\ Instituto de Automática Industrial, CSIC. \\ Ctra. Campo Real km 0.200 - La Poveda. \\ 28500 Arganda del Rey, Madrid, Spain. \\ iglesias@iai.csic.es \\ lola@,iai.csic.es \\ nachosm@iai.csic.es \\ joliva@,iai.csic.es
}

\begin{abstract}
A situation consisting in evaluating and choosing among alternative actions can be managed from the point of view of Decision Making (DM). This paper presents an approach to design and develop Decision Support Systems (DSS) to be applied in emergency situations. In these situations the decision maker is under heavy stress because each different decision implies different important outcomes related with human and economic losses. First of all, a domain knowledge base has to be built from both the properties of emergency situations and the actions devoted to counteract them. From this knowledge, three different DM methods, based on the Probability Theory and the Possibility Theory, process the incoming emergency information and choose the best action for putting out the emergency situation. The resulting decisions of each method over a set of plausible emergency situations can be evaluated by a domain expert and the method with the best average performance can be built in the DSS. This DSS can help a decision maker find out an optimal decision in a short period of time maximizing security and minimizing stress.
\end{abstract}

\section{Introduction}

Making a decision means choosing among the possible alternatives or courses of action to solve a problem which one satisfies the proposed objectives according to a certain preference order. The objectives indicate the desirable values that the parameters or criteria of the context of the problem should reach. DM methods evaluate alternatives with respect to objectives, i.e. they analyze outcomes of each alternative or decision evaluating whether the values of the criteria satisfy the objectives and assigning the corresponding score to each alternative. The best ranked alternative should be the optimal decision to be made.

There are different contexts where DM can be applied. Typical financial problems like choosing whether to buy or sell stocks are purely economic [17]. In classical health diagnoses [2], doctors can take as long as they need to find out a solution matching patients' symptoms and analyzed parameters. Other problems, like choosing the best means of transport [11] to make a trip does not involve any time or economic loss requirements. Decisions in emergency situations are hard decisions that must be made quickly because their consequences could be catastrophic. Emergency situations are sudden, urgent, usually unexpected incidents requiring immediate action which often involve danger. DM in emergency situations is an interesting field of study because the stress exerted on decision makers does not allow them to think clearly and make optimal decisions, so an automatic system would aid them keep security levels. Systems that help a decision maker make better decisions providing 
assistance for specific decision making tasks are called Decision Support Systems (DSS), which were first coined by [6], [12]. There are a lot of situations that can be classified into emergencies but there is scarce literature about managing these situations from a computational point of view. Emergency management of nuclear accidents using a DSS is presented in [8]. Another DSS described in [1] aids strategic planning and policy making for disaster mitigation. Concretely, it is designed for earthquake scenarios, estimating the extent of human losses and injuries.

An important feature of making a decision in whichever context is the uncertainty produced by a lack of information about the decisions and their consequences. In order to model uncertainty, there are different parametric methods for making a decision [5]. These methods can help a decision maker find out an optimal decision in a short period of time by minimizing stress. On the one hand, there are methods from the Probability Theory which represent DM problems using influence diagrams [9]. On the other hand, there are methods from the Possibility Theory which are based on fuzzy logic and fuzzy sets [18]. The Probability Theory provides a quantitative description of the likelihood of a particular event. The Possibility Theory manages events that belong to fuzzy sets with a certain degree of membership. DM methods based on possibility measures allow to formalize uncertain, imprecise concepts inherent in decision making problems.

This paper deals with a methodology to represent the domain knowledge and automatically making a decision in emergency situations. Due to the novelty of the application domain and the lack of real computational systems for it, a comparative study of both possibility and probability DM methods is carried out in order to choose the method that performs the best when the DSS manages a fire that starts in the ground floor of a building. This is a very common emergency that implies a decrease in the security of the building and its occupants. The main menaces for building occupants are smoke and toxic gases as well as heat and flames [4]. Because of the security decrease, the objectives that the different possible decisions must fulfill are maximizing different levels of safety so that the building can restore security after the emergency. The hybrid nature of the methodology is supported by the combination of the problem representation, based on the possibility theory, with the methods inferring decisions, based on the possibility theory as well as the probability theory.

\section{DSS knowledge base for an emergency}

A DSS needs a knowledge base containing the criteria or parameters of the emergency and the way to automatically tackle it. In order to make an optimal decision, the alternatives available to maximize security have to be compared by evaluating the way the alternatives modify the criteria to fulfill the objectives. These criteria are security features of the environment and, depending on the selected alternative, their values at the beginning of the emergency can be modified through the environmental changes associated to the alternative. In the simple fire emergency discussed in this paper, three criteria are considered: 'Cost', which is the cost that the fire could cause in economic terms; 'Spread', which describes the fire spread and it decreases as the control over the fire is increased; and 'Risk', which represents the level of risk that may be reached by the building occupants.

In order to represent the environmental model and the expert knowledge about the criteria involved in an emergency situation, an artificial weighted net of concepts has been generated. This net provides an intuitive way to describe the properties associated to a certain emergency and their relations. A net concept stands for a criterion or an event whose value may depend on the values of other different events. If concept A depends on concept B they are associated by adding a directed connection from $\mathrm{B}$ to $\mathrm{A}$. The association weights between net concepts are considered here as a level of influence of the origin concept on the end concept.

The setting of the correct net concepts, their connections as well as the association weights has to be carried out by a knowledge engineering process that allows to translate the expert knowledge into the conceptual net. For instance, the following piece of domain knowledge could be represented as shown in Figure 1: the toxicity of a fire is calculated using the amount of smoke released that on its part depends, among other things, on the speed of fire spread and the material which is burning. The criterion called 'Risk' is dependant to a greater extent on the toxicity of the fire than on the heat. The values of the parameters that contribute to the criteria can be obtained from sensors or online measurements (Heat), data bases (Material) or computed by different procedures (Speed).

This net of concepts can be seen as Bayesian network since it is a way to represent causal knowledge [14] which does not include decision nodes and so it can not be used to infer their values. 


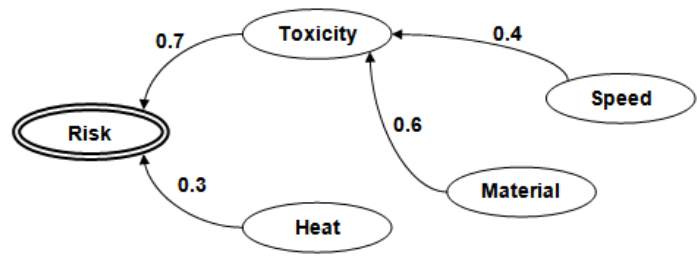

Figure 1. Weights and connections of the criterion 'Risk'.

There are several actions for controlling fire [13] but, for the sake of simplicity, this paper considers only two of them: evacuating people or extinguishing the fire by using local resources. The alternatives shall be associated to these actions and, besides their own operative procedure, they could include deterministic actions, i.e., advice to firemen and police, but this issue is out of the scope of this paper. So, the alternatives here considered are three: evacuating building occupants, extinguishing the fire using a fire extinguisher or extinguishing the fire using a sprinkler system. The 'Evacuate' alternative will decrease the 'Risk' criterion very much, but it will keep unchangeable the 'Cost' and the 'Spread' criteria. Each alternative can satisfy different objectives that express the desirable value the criteria must take. The alternative that fulfils the objectives will be the best.

Given a decision, it is difficult to predict how the environment will evolve and how any decision would affect the environment, i.e. what consequences would be produced over the environment and hence, over the values of the criteria. This evolution of the environmental conditions must be also included in the knowledge base. In the example presented in this paper, this knowledge has been represented by functions that symbolize how each alternative modifies the current values of criteria as shown in Table 1 for the 'Cost' and 'Risk' criteria.

Table 1. Evolution functions.

\begin{tabular}{|c|c|}
\hline & Cost \\
\hline Sprinkler & $f_{\text {Sprinkler }}$ (Cost) $=0.5 \cdot$ Cost \\
\hline Evacuate & $f_{\text {Evacuate }}($ Cost $)=1.0 \cdot$ Cost \\
\hline Extinguisher & $f_{\text {Extinguisher }}($ Cost $)=0.3 \cdot$ Cost \\
\hline & Risk \\
\hline Sprinkler & $f_{\text {Sprinkler }}$ (Risk) $)=0.7 \cdot$ Risk \\
\hline Evacuate & $f_{\text {Evacuate }}($ Risk $)=0.1 \cdot$ Risk \\
\hline Extinguisher & $f_{\text {Extinguisher }}($ Risk $)=0.8 \cdot$ Risk \\
\hline
\end{tabular}

Because each alternative satisfies different objectives, the best alternative for solving each emergency problem depends on the objectives and their preference order, both of them determined by the decision maker. The objective handled in the fire emergency is minimizing the values of each criterion. The predicted values of each criterion after applying the evolution functions are weighted by their corresponding preference.

\section{DSS methods for evaluating alternatives}

The form of processing all the information and data coming from the environment differs from one DM method to another. The next sections present the three methods introduced in Section 1.

\subsection{General fuzzy method}

The general fuzzy method [16] requires criteria represented as triangular fuzzy numbers. A triangular fuzzy number $\tilde{a}$ is defined by a triplet $\left(a_{1}, a_{2}, a_{3}\right)$ where $a_{1}$ is the minimum possible value of a criterion, $a_{2}$ is the most possible value and $a_{3}$ is the maximum possible value. Accordingly, the values calculated for each criterion from the concept net are modified depending on its evolution function and transformed into triangular fuzzy numbers. Fuzzy sets used by the fuzzy methods are built from expert knowledge.

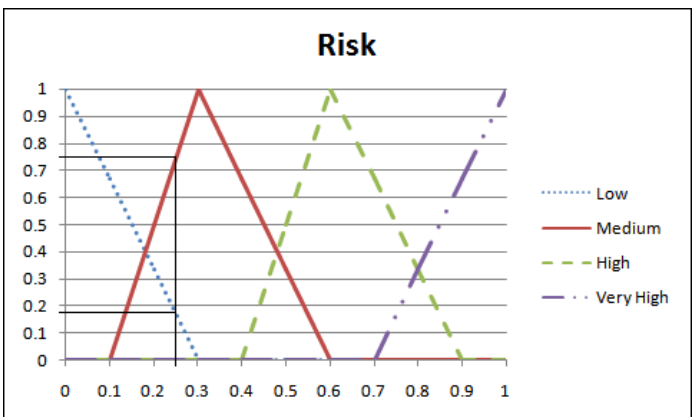

\section{Figure 2. Fuzzy sets representing the possible values of criterion 'Risk'.}

For instance, as shown in Figure 2, for the fire emergency, if the value predicted by the evolution function of an alternative for the 'Risk' criterion is 0.25 , then its final value is transformed into the fuzzy number $(0.1,0.25,0.6)$ using the corresponding fuzzy set. Since the value is 0.25 , the degree of membership of the 'Low' set (0.17) is shorter than of the 'Medium' set (0.75). Therefore, the anchors of the 'Medium' set $(0.1$ and 0.6$)$ are chosen as the triplet's bounds. Once these fuzzy numbers have been calculated, the method uses the vertex method [3] to calculate the performance of each alternative with regard to ideal solutions. The most preferred alternative must have the shortest 
distance from the positive ideal solution and the longest distance from the negative ideal solution.

\subsection{Fuzzy method based on eigenvector}

This method uses trapezoidal fuzzy numbers that are represented by four terms. A trapezoidal fuzzy number $\tilde{a}$ is defined by a quartet $\left(a_{1}, a_{2}, a_{3}, a_{4}\right)$ whose meaning is similar to triangular fuzzy numbers. Notice that if $a_{2}=a_{3}$ then $\tilde{a}$ is a triangular fuzzy number. First of all, the method constructs a matrix where rows are alternatives and columns are criteria. Each matrix cell contains a trapezoidal fuzzy number that is the value of an alternative with respect to a criterion. This matrix is called fuzzy attribute evaluation matrix. Once the matrix is constructed, the method computes a centrally normalized matrix from the first one and then transforms it into a symmetry matrix, regarding to covariance definition of random variables. Finally, the method determines the eigenvectors of the last matrix which are used as the alternatives' performance. Thus, the eigenvector contains a value for each alternative and the most preferred alternative must have the highest value. The details of this method are widely described in [7].

\subsection{Influence diagrams}

This method needs a utility function that measures the fitness of any possible decision outcome. In order to compute the best decision, it is necessary to combine the utility function with knowledge of how the decisions affect outcomes, i.e. the subjective probability. The expected utility is obtained by equation (1) and it represents the alternatives' performance. Therefore, the most preferred alternative is defined as the one that will maximize the expected utility (for more details on utility theory see [15]). If the criterion is a benefit one, then its utility is calculated using equation (2). If it is a cost criterion, then its utility is calculated using equation (3), where $X$ represents the value of such criterion.

$$
\begin{gathered}
E[\text { Utility(decision) }]=\sum_{\begin{array}{c}
\text { possible } \\
\text { outcomes }
\end{array}} p(\text { outcome |decision) } \cdot U(\text { outcome }) \\
U_{i}=1-\frac{X_{\max }-X_{i}}{X_{\max }-X_{\min }} \\
U_{i}=\frac{X_{\max }-X_{i}}{X_{\max }-X_{\min }}
\end{gathered}
$$

The method based on influence diagrams needs to assign to all possible outcomes their corresponding probabilities and to define the utility functions using expert knowledge.

\section{Updating the concept net}

When an emergency is detected, the DSS evaluates the environment so the values of the parameters contained in the weighted net are automatically updated. Then, the net calculates the values of the different criteria. At this point, before the DSS applies the DM methods for computing the score of each alternative, it calculates the criteria's evolution and transforms the resulting discrete values into triplets using fuzzy sets, as described in Section 3.1 and shown in Figure 3.

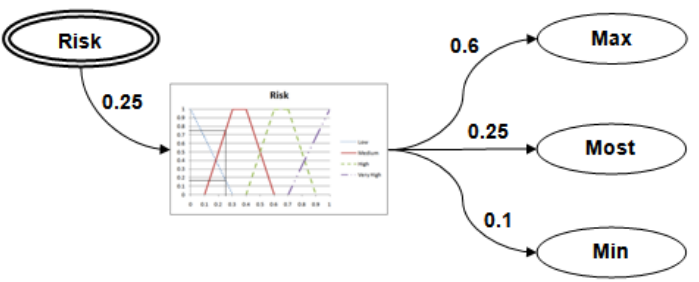

\section{Figure 3. Obtaining the maximum, most and minimum possible values of 'Risk'.}

Once the DSS has obtained these values, it builds a representation of the problem suitable for applying the corresponding DM method as described in Figure 4. If it uses the general fuzzy method, then the possible values are organized in triplets. If it uses the eigenvector, then the most possible value is duplicated in order to construct trapezoidal fuzzy numbers. The possible outcomes of a decision are calculated from the fuzzy triplet (max, most, $\min$ ).

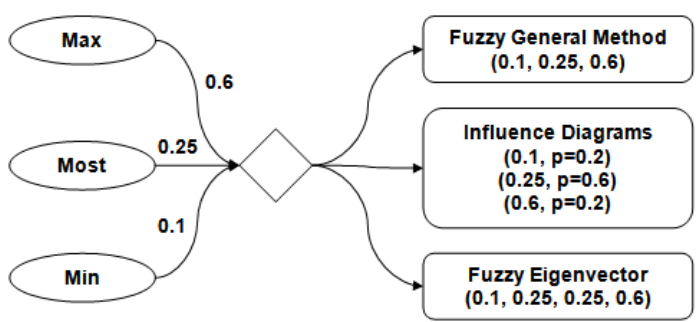

Figure 4. Organizing the values depending on the method that must be applied.

Although this fuzzy transformation belongs to the Possibility Theory, it can be used to obtain a discrete value of a criterion from its continuous one, making the management of the problem homogeneous for the three methods. After that, every criterion has three 
possible values so there are twenty seven possible outcomes (combination of the three values of the three criteria) for the decision making method using influence diagrams. This issue gives a hybrid nature to this method.

\section{Empirical evaluation}

The experiment performed consists in the evaluation of the three methods exposed above on seven different situations where the fire emergency is the same but the decision maker preferences change. Every situation uses the same emergency environment in which the values computed by the weighted net for the 'Cost', 'Spread' and 'Risk' criteria are 0.4, 0.6 and 0.6 , respectively. The values of each criterion regarding each alternative are modified depending on evolution functions. When dealing with influence diagrams, the probability assigned to a criterion that has the minimum or maximum possible value is 0.2 and the probability for a criterion that has the most possible value is 0.6 . Table 2 shows the empirical results obtained, where the preferences are shown in the second column, and they represent the order for fulfilling the objective of minimizing the 'Cost', 'Spread' and 'Risk' criteria. The remaining columns contain the ranking of the alternatives using the different DM methods, which are ordered according to their score. Boldface indicates the best alternative.

Table 2. Ranking of alternatives.

\begin{tabular}{|c|c|c|c|c|}
\hline & Preferences & $\begin{array}{c}\text { Fuzzy } \\
\text { General } \\
\text { Method }\end{array}$ & $\begin{array}{c}\text { Fuzzy } \\
\text { Eigenvector }\end{array}$ & $\begin{array}{l}\text { Influence } \\
\text { Diagrams }\end{array}$ \\
\hline 1 & $(0.33,0.33,0.33)$ & $\begin{array}{c}\text { Sprinkler } \\
\text { Extinguisher } \\
\text { Evacuate }\end{array}$ & $\begin{array}{c}\text { Sprinkler } \\
\text { Extinguisher } \\
\text { Evacuate }\end{array}$ & $\begin{array}{l}\text { Sprinkler } \\
\text { Evacuate } \\
\text { Extinguisher }\end{array}$ \\
\hline 2 & $(0.5,0.3,0.2)$ & $\begin{array}{l}\text { Sprinkler } \\
\text { Extinguisher } \\
\text { Evacuate }\end{array}$ & $\begin{array}{l}\text { Sprinkler } \\
\text { Extinguisher } \\
\text { Evacuate } \\
\end{array}$ & $\begin{array}{l}\text { Sprinkler } \\
\text { Extinguisher } \\
\text { Evacuate }\end{array}$ \\
\hline 3 & $(0.5,0.2,0.3)$ & $\begin{array}{l}\text { Sprinkler } \\
\text { Extinguisher } \\
\text { Evacuate }\end{array}$ & $\begin{array}{l}\text { Sprinkler } \\
\text { Extinguisher } \\
\text { Evacuate } \\
\end{array}$ & $\begin{array}{c}\text { Sprinkler } \\
\text { Evacuate } \\
\text { Extinguisher }\end{array}$ \\
\hline 4 & $(0.3,0.5,0.2)$ & $\begin{array}{l}\text { Sprinkler } \\
\text { Extinguisher } \\
\text { Evacuate } \\
\end{array}$ & $\begin{array}{l}\text { Sprinkler } \\
\text { Extinguisher } \\
\text { Evacuate } \\
\end{array}$ & $\begin{array}{l}\text { Sprinkler } \\
\text { Extinguisher } \\
\text { Evacuate }\end{array}$ \\
\hline 5 & $(0.3,0.2,0.5)$ & $\begin{array}{c}\text { Sprinkler } \\
\text { Evacuate } \\
\text { Extinguisher }\end{array}$ & $\begin{array}{c}\text { Sprinkler } \\
\text { Evacuate } \\
\text { Extinguisher }\end{array}$ & $\begin{array}{c}\text { Evacuate } \\
\text { Sprinkler } \\
\text { Extinguisher }\end{array}$ \\
\hline 6 & $(0.2,0.5,0.3)$ & $\begin{array}{l}\text { Sprinkler } \\
\text { Extinguisher } \\
\text { Evacuate }\end{array}$ & $\begin{array}{l}\text { Sprinkler } \\
\text { Extinguisher } \\
\text { Evacuate } \\
\end{array}$ & $\begin{array}{l}\text { Sprinkler } \\
\text { Extinguisher } \\
\text { Evacuate }\end{array}$ \\
\hline 7 & $(0.2,0.3,0.5)$ & $\begin{array}{c}\text { Sprinkler } \\
\text { Evacuate } \\
\text { Extinguisher }\end{array}$ & $\begin{array}{l}\text { Sprinkler } \\
\text { Extinguisher } \\
\text { Evacuate } \\
\end{array}$ & $\begin{array}{c}\text { Evacuate } \\
\text { Sprinkler } \\
\text { Extinguisher }\end{array}$ \\
\hline
\end{tabular}

There are some interesting remarks about Table 2. The first one is that both fuzzy methods rank better 'Sprinkler' than the other two alternatives in all situations. This result does not seem to be realistic because the preferences are different. However, the fuzzy general method is more reliable than the fuzzy method based on eigenvector because in the two situations where 'Risk' is the most preferred criterion, i.e. the main goal is to minimize 'Risk' that has a preference of 0.5, 'Evacuate' has a higher score than 'Extinguisher' (see rows 5 and 7 in Table 2). This last alternative should be the most appropriate one when 'Risk' is the most important criterion because it decreases 'Risk' very much. The last remark is that influence diagrams model the preferences better than the other two methods. This assessment is derived from the situations where 'Risk' is the most important criterion. In these situations, influence diagrams place 'Evacuate' with the best score, which must be the most suitable alternative. In contrast, the fuzzy method is faster than the Influence Diagram method because the fuzzy method needs less calculations for each decision, although the difference is worthless. Summarizing, the method based on influence diagrams seems to be the most appropriate one for the problem presented in this paper because the best ranked alternative obtained by this method complies with the preferences better than the other methods.

According to these empirical results, the DSS for fire emergency should be implemented using the method based on influence diagrams which seems to be the most suitable for the fire emergency providing a worthy outcome.

Despite of the efficiency and the comprehensible way to express the expert knowledge of the methods based on possibility and the fact that influence diagrams need a greater amount of expert knowledge, influence diagrams make a better decision in the emergency situation considered here regarding the preferences of the decision maker.

\section{Conclusions}

The methodology presented in this paper allows to design and develop decision support systems for emergency. This DSS can help a decision maker find out a suitable decision in a short period of time maximizing security and minimizing stress by automatically analyzing all relevant information and pointing out the best alternative. In these situations, the decision maker cannot afford the large amount of data that must be considered and the DSS aids him/her in finding out a decision. Decision makers find very difficult to trust in systems which have strange or unintelligible operating ways. The proposed 
methodology provides a comprehensible supervision for systematic thinking in emergency situations.

In order to build an effective and useful DSS, it must contain a complete and consistent knowledge base representing the main features of an emergency situation, involving net weights, and the interaction between alternatives and the environment, including evolution functions. This knowledge base is a valuable structure to make a decision and it has to be collected carefully in order to model the problem as real as possible. In the fire emergency, a greater number of well defined alternatives could be added to the knowledge base in order to concrete details about what extinguisher is the best one to use and where it is, or how people should be evacuated and the best path to do that. Emergency DSSs are almost impossible to prove in real situations because emergencies are sudden incidents and they are very difficult to predict. Therefore, the only way to check whether it works well is doing simulations of hypothetic emergency situations. A history of emergency situations would be a very valuable source to compute the weights of the net and the decision maker preferences. Using a historical data base, it would make possible to check whether the DSS can learn from past mistakes comparing the results obtained by the DSS and the ones obtained by well trained experts, for instance, firemen. Using this expert knowledge it would be possible to invert the decision problem to infer which priors, likelihoods and utility functions firemen used to make their decisions [10].

\section{References}

[1] F. Aleskerov, A.I. Say, A. Toker, H.L. Akin, and G.A. Altay, "Cluster-Based Decision Support System for Estimating Earthquake Damage and Casualties", Disasters, 29(3), 2005, pp. 255-276.

[2] A.T. Bahill, K. Bharathan, and R.F. Curlee, "How the Testing Techniques for a Decision Support System Changed Over Nine Years", IEEE Transactions on Systems, Man, and Cybernetics, 25(12), 1995 pp. 1533-1542.

[3] C.T. Chen, "Extensions of the TOPSIS for Group Decision-making Under Fuzzy Environment", Fuzzy Sets and Systems, 114, 2000, pp. 1-9.

[4] A. Emsley, A. Lim, and G. Stevens, "International Fire Statistics and the Potential Benefits of Fire CounterMeasures", Polymer Research Centre Report, University of Surrey, Guilford, Surrey GU2 7XH, UK, 18 May 2005.

[5] J. Figueira, S. Greco, and M. Ehrgott, M. (eds), Multiple Criteria Decision Analysis: State of the Art Surveys, Springer Science + Business Media, Inc: Boston, 2005.
[6] A. Gorry, and M. Scott-Morton, "A Framework for Management Information Systems", Sloan Management Review, 13(1), 1971, pp. 55-70.

[7] X. Gu, and Q. Zhu, "Fuzzy Multi-attribute Decisionmaking Method Based on Eigenvector of Fuzzy Attribute Evaluation Space", Decision Support Systems, 41(2), 2006, pp. 400-410.

[8] R.P. Hämäläinen, M.R.K. Lindstedt, and K. Sinkko, "Multiattribute Risk Analysis in Nuclear Emergency Management", Risk Analysis, 20(4), 2000, pp. 455-467.

[9] R.A. Howard, and J.E. Matheson, "Influence Diagrams", Decision Analysis, 2(3), 2005, pp. 127-143.

[10] K. Körding, "Decision Theory: What "Should" the Nervous System Do?”, Science, 318(5850), 2007, pp. 606610

[11] A., Iglesias, M.D. del Castillo, M. Santos, and J.I. Serrano, "Control para la Toma de Decisiones: Estudio Comparativo de Técnicas Inteligentes", Actas de las XXVIII Jornadas de Automática, Universidad de Huelva, Huelva. 2007.

[12] M. Mora, G.A. Forgionne, and J.N.D. Gupta (eds), Decision Making Support Systems: Achievements, Trends and Challenges for the New Decade. Idea Group Inc: London, 2003.

[13] S.K. Ray, and R.P. Singh, "Recent Developments and Practices to Control Fire in Underground Coal Mines", Fire Technology, 43(4), 2007, pp. 285-300.

[14] S. Russell, and P. Norvig, Artificial Intelligence: A Modern Approach, Englewood Cliffs, NJ: Prentice-Hall, 1995.

[15] J. Von Neumann, and O. Morgenstern, Theory of Games and Economic Behavior (Commemorative Edition). Princeton University Press, Princeton, 2004.

[16] W. Wang, and N. Fenton, "Risk and Confidence Analysis for Fuzzy Multicriteria Decision Making", Knowledge Based Systems, 19, 2006, pp. 430-437.

[17] W. Yan, and C.D. Clack, "Evolving Robust GP Solutions for Hedge Fund Stock Selection in Emerging Markets", Proc. of the $9^{\text {th }}$ annual conference on Genetic and evolutionary computation, eds. D. Thierens, H. G. Beyer \& J. Bongard, ACM Press, volume 2, London, 2007, pp. 22342241.

[18] L. Zadeh, "Fuzzy Sets as the Basis for a Theory of Possibility", Fuzzy Sets and Systems, 1, 1978, pp. 3-28. 